Cipango Cahiers d'études japonaises

$20 \mid 2013$

Nouveaux regards sur les arts de la scène japonais I

Michael Lucken, Les Japonais et la guerre, 1937-1952

Paris, Fayard, 2013, 400 p. Prix Thiers de l'Académie française 2014

Arnaud Doglia

(2) OpenEdition

Journals

Édition électronique

URL : https://journals.openedition.org/cipango/1978

DOI : 10.4000/cipango.1978

ISSN : 2260-7706

Éditeur

INALCO

Édition imprimée

Date de publication : 30 octobre 2013

ISSN : 1164-5857

Référence électronique

Arnaud Doglia, « Michael Lucken, Les Japonais et la guerre, 1937-1952 », Cipango [En ligne], 20 | 2013 ,

mis en ligne le 26 novembre 2014, consulté le 30 juin 2021. URL : http://journals.openedition.org/

cipango/1978; DOI : https://doi.org/10.4000/cipango.1978

Ce document a été généré automatiquement le 30 juin 2021.

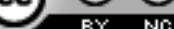

Cipango est mis à disposition selon les termes de la Licence Creative Commons Attribution - Pas d'Utilisation Commerciale 4.0 International. 


\title{
Michael Lucken, Les Japonais et la
} guerre, 1937-1952

\author{
Paris, Fayard, 2013, 400 p. Prix Thiers de l'Académie française 2014
}

\section{Arnaud Doglia}

1 À l'heure où les relations entre le Japon et ses voisins sont au centre de querelles historiques (et politiques) fortement médiatisées, l'ouvrage de Michael Lucken tombe à point nommé. On prendra pour exemple un cliché du Premier ministre Abe Shinzō, souriant et installé dans le cockpit d'un avion d'entraînement des forces d'autodéfense japonaises, avec peint sur le fuselage de l'appareil le numéro « 731 ». Il n'en fallait pas plus à une partie de la presse coréenne (et asiatique) pour transformer la photo en scandale, et rappeler les atrocités médicales et scientifiques dont fut responsable l'Unité 731 sur le continent entre les années 1930 et 1945, avant d'enchaîner sur la question des «femmes de réconfort » et du système de prostitution institutionnalisée par Tōkyō pendant la guerre.

2 S'il est vrai que la majorité de ces esclaves sexuelles étaient de nationalité coréenne, et que la question représente un problème majeur dans les relations entre la Corée du Sud et le Japon, il n'en va pas de même pour l'Unité 731. Non seulement, car les victimes de l'arme biologique japonaises furent en grande majorité des citoyens chinois, mais aussi, car outre la responsabilité de l'État, la question des « femmes de réconfort » ne partage pas de point commun avec la mise en place d'un programme de guerre bactériologique et chimique. Il en va de même au niveau de la mémoire de ces événements. La question de l'esclavage sexuel de ces femmes reste un enjeu mémoriel sensible, alors que le sujet de l'arme biologique n'est plus discuté par les révisionnistes et les nationalistes depuis la moitié des années 1980. Autant d'amalgames et d'erreurs qui viennent s'ajouter à un autre lieu commun: celui d'un Japon présenté trop souvent comme une entité monolithique, incapable de repentir, et en déni total face aux atrocités commises jusqu'en 1945. Pour le dire autrement, le Japon aurait un " problème de mémoire ».

3 Les Japonais et la guerre s'attaquent donc à un sujet sensible et terriblement actuel, afin d'aider le lecteur francophone à reconsidérer l'histoire d'un pays éloigné à la fois de ses préoccupations géographiques et culturelles. Divisé en onze sections, il offre un 
panorama à la fois clair et détaillé de ce que représente la Seconde Guerre mondiale pour les Japonais. Après une introduction présentant le but de l'ouvrage ( mettre en évidence les interactions fécondes entre l'histoire et la culture ", p.13), le premier chapitre, intitulé "La nation en conquête ", propose naturellement une présentation de l'arrière-plan historique des années 1930 sous différents aspects, une dynamique récurrente à travers tout l'ouvrage. L'archipel est ainsi abordé sous l'angle des médias, des représentations de l'autre, du peuple, du tourisme, des pratiques funéraires, des arts, ou encore du tourisme, pour n'en citer que quelques-uns. Cette abondance de perspectives permet de mettre en lumière de manière variée et agréable à la lecture une des thèses centrales de l'auteur : l'impossibilité de résumer le Japon à un seul bloc, de par la multitude des expériences de chacun. Michael Lucken nous rappelle au passage que contrairement à l'idée selon laquelle Tōkyō visait la domination mondiale, idée centrale aux États-Unis pendant et après la guerre, « le Japon ne semble pas avoir eu de plans à long terme [...] ni de programme politique clair et unifié » (p. 33).

Le second chapitre, "Une dynamique totalitaire", débute par une discussion sur la définition du concept de totalitarisme. L'auteur y présente les points de vue japonais et occidentaux sur une question qui continue de hanter les spécialistes de la période : le Japon fut-il fasciste? L'intérêt principal de cette partie est de montrer qu'il existe un réel débat à ce propos, et que vue de l'Occident, la question n'est pas aussi simple que l'on aimerait se l'imaginer. Ainsi, un mouvement d'unité nationale tel que l'«Association de soutien au trône » ne peut être comparé ni au Parti communiste en URSS ni au Parti nazi en Allemagne. Là où trop d'auteurs collent au Japon des années 1940 l'étiquette fasciste, Michael Lucken démontre clairement le besoin de nuancer la perspective.

Le troisième chapitre, "Le sens du combat », est dans un premier temps consacré à une analyse de la guerre, dans sa définition comme sur les champs de bataille. Fidèle à sa volonté de considérer la perspective des artistes durant le conflit, l'auteur s'interroge sur la manière dont ceux-ci perçoivent la guerre de leur vivant, lui donnent un sens et la justifient. Ce point de vue l'amène à discuter également de l'appellation du conflit, un sujet naturellement traité par les historiens japonais, mais qu'il est heureux de voir ici rendu accessible au lecteur qui ne maîtrise pas la langue japonaise. Ce qui se conçoit en Occident comme la Seconde Guerre mondiale peut, au Japon, être constitué d'« incidents » (jiken) et d'« événements " (jihen), une perspective que Michael Lucken démontre en s'appuyant autant sur les écrits de philosophes que d'historiens ou de critiques littéraires.

Dans une seconde partie, dédiée au conflit lui-même, il attire notre attention sur des atrocités de guerre peu connues en Europe, comme l'emploi de l'arme chimique sur le continent, ou encore les massacres en Asie du Sud-Est. L'auteur présente de manière claire la dichotomie entre le discours officiel japonais, qui parle de libération des peuples asiatiques, et la réalité des atrocités perpétrées par l'armée impériale. Notons également que si Michael Lucken reprend le travail de l'historien étasunien John Dower sur les perceptions américaines et japonaises de l'« Autre » et le racisme évident qui en découle, il s'intéresse également à la " question juive » en Asie, une initiative originale qui contribue à faire de cet ouvrage un excellent panorama général de la période.

Dans un quatrième chapitre intitulé "Les héros et les morts", l'auteur centre son propos sur le rôle de l'empereur durant comme après la guerre. S'appuyant sur les recherches de grands historiens japonais comme Awaya Kentarō, Michael Lucken 
démontre non seulement que l'idée selon laquelle les Japonais n'auraient jamais fait face à leurs responsabilités de guerre est fausse, mais également que l'empereur n'est pas comparable aux souverains occidentaux, notamment grâce à l'efficacité des structures politiques en place dès la fin du $\mathrm{XIX}^{\mathrm{e}}$ siècle, et l'utilisation des médias pour renforcer le pouvoir impérial au cours du $\mathrm{xx}^{\mathrm{e}}$ siècle. Ce raisonnement est prolongé par une analyse du sanctuaire du Yasukuni, lieu central lorsqu'est abordée la question des responsabilités impériales. Or, comme dans le reste de l'ouvrage, l'intérêt principal réside dans son originalité : l'auteur ne s'arrête pas à l'étude d'un seul lieu, mais présente la mise en place durant le $\mathrm{xx}^{\mathrm{e}}$ siècle d'un réseau de sanctuaires, de stèles et d'ossuaires à travers le pays afin de consolider l'idéologie nationale et la fidélité à l'empereur. L'analyse de ces derniers lieux est particulièrement bienvenue, en ce qu'elle permet, sans jamais sombrer dans le culturalisme, de mettre en avant les différences de pratiques funéraires entre les Japonais et les Américains sur le champ de bataille.

8 Suite logique de la question du traitement des corps et des ossements, le chapitre «Peur et destruction » est notamment consacré aux bombardements alliés sur le Japon, ainsi qu'aux kamikazes et à la théâtralisation de la défaite. L'auteur montre clairement en quoi la menace venue du ciel s'impose aux Japonais comme une source d'angoisse et de traumatisme, posant les bases d'un pays "victime», une perspective encore aujourd'hui au cœur du débat sur les mémoires de guerre dans l'archipel. À travers cette analyse de la propagande, du contrôle des images et de la naissance du mythe du héros et de son sacrifice, le lecteur prend mieux conscience du quotidien de la population japonaise jusqu'à la fin de la guerre.

9 Le chapitre suivant, "Les coordonnées complexes de l'après-guerre ", propose un décryptage de la mythologie du 15 août 1945, journée symbolique de la défaite, mais aussi du maintien de l'empereur sur le trône. Ce débat historiographique bien connu au Japon est pour la première fois présenté en langue française, afin de montrer comme Hirohito fût transformé en une figure bienveillante, débarrassé (temporairement) de ses responsabilités de guerre. Ce chapitre est également l'occasion d'expliciter plus en détail les dates choisies dans son titre (1937-1952) pour parler de la guerre. 1952 représente en effet une année phare, allant de la fin de l'occupation militaire du Japon à la signature de traités internationaux, qui amène Michael Lucken à faire durer « la guerre " au-delà de 1945. Cette perspective originale est la bienvenue en ce qu'elle permet de considérer non seulement le Japon, mais également ses relations avec les pays voisins. La question des manuels scolaires et de l'écriture d'une histoire conjointe (Chine, Japon, Corée du Sud) est également brièvement traitée, montrant que l'histoire de l'archipel doit à juste titre être intégrée à une réflexion qui dépasse le cadre national.

10 Une fois la fin de la guerre advenue, il convient de se pencher sur ses conséquences. Dans «L'occupation américaine, ou le présent contre le passé », l'ouvrage traite de la propagande "démocratique » américaine, ainsi que du démantèlement des structures culturelles et idéologiques du Japon impérial. Plutôt qu'un discours classique sur l'abolition du shintō d'État, l'auteur s'intéresse à celui qui fut responsable de cette politique, l'Américain William K. Bunce. Là encore, cette approche est particulièrement intéressante dans la mesure où, à l'inverse des livres d'histoire classiques, elle permet de mettre sous les projecteurs des personnages ayant joué un rôle important dans l'occupation du Japon, sans pour autant que leurs noms soient restés dans l'histoire (on 
retiendra notamment ici Charles Willoughby, Courtney Whitney ou Bonner Fellers). La question du Procès de Tōkyō et des responsabilités impériales est également discutée avec finesse, et les nombreuses citations venant autant du peuple que des intellectuels ou des anciens militaires contribuent à rendre le texte vivant et plaisant. Enfin, une analyse de la transformation des monuments en des symboles de paix à travers le pays complète ce panorama de la période d'occupation.

11 Le huitième chapitre, "L'histoire au pluriel », revient sur une question centrale pour l'auteur: la pluralité des points de vue et des expériences de guerre. À travers une présentation des manuels scolaires japonais et de l'historiographie américaine, il devient clair que le Japon d'après 1945 est une construction commune aux Alliés et aux Japonais, loin du mythe de la seule domination du General Headquarters (GHQ). Michael Lucken mentionne également à juste titre les premières parutions dans l'archipel de confessions des crimes de guerre, contribuant ainsi à détruire le mythe d'un Japon incapable de repentir et amnésique.

12 Comme son titre l'indique, le chapitre "Conscience individuelle et inertie collective " s'attache à diviser les Japonais qui ont connu la guerre en quatre groupes distincts. L'intérêt de la démarche permet d'insister sur la continuité des mondes politiques et scientifiques avant comme après la guerre (filiation des politiciens, prolongation de carrière pour les scientifiques intégrés au programme d'armement biologique japonais jusqu'en 1945). Michael Lucken élargit ensuite son panorama de la période en s'appuyant sur l'excellent travail d'Igarashi Yoshikuni ${ }^{1}$ sur le sport et la culture populaire durant et après l'occupation, afin de souligner l'importance du dépassement physique de soi comme remède au traumatisme de la défaite. Afin de replacer le Japon dans son contexte, l'auteur discute ensuite des excuses offertes par Tōkyō à ses voisins, et après avoir dressé des parallèles avec la situation en Europe, en arrive à une conclusion tout à fait à propos: "Pour des raisons en partie géopolitiques, les États coréens, chinois et japonais n'ont pas su créer les conditions d'un rapprochement des mémoires ; au contraire, ils se sont engagés [...] sur le chemin de la confrontation, en dépit du développement des collaborations scientifiques et culturelles» (p. 256).

Le dixième chapitre, " Mémoire et religion ", prend la forme d'une suite à la discussion lancée auparavant sur le sanctuaire du Yasukuni. Michael Lucken y présente notamment un autre monument tout aussi important, mais trop souvent ignoré, le mémorial de Chidorigafuchi. L'examen de sa structure comme de sa fonction permet à l'auteur de continuer à analyser le rôle des ossements et du traitement des corps, à travers l'engagement d'associations citoyennes à la recherche de dépouilles sur les vestiges de champs de bataille en Asie. Ce chapitre très instructif et original s'intègre parfaitement au reste de l'ouvrage. Notons par exemple l'analyse de la transformation après la guerre de la statue d'un soldat en celle d'un "bodhisattva compatissant " (p. 284), parfait exemple s'il en est de l'évolution des discours et des consciences historiques dans l'archipel.

14 Le dernier chapitre, "Du monument au musée : le chemin difficile de l'apaisement ", élargit le propos de la partie précédente en revenant notamment sur les exemples de Nagasaki et Hiroshima. En se basant sur des sources japonaises comme américaines, l'auteur montre que derrière un style qui se veut analytique, le véritable discours sur l'arme atomique est avant tout destiné à susciter l'effroi et faire jouer les émotions. Cette analyse des discours n'est d'ailleurs pas uniquement limitée aux monuments, mais est appliquée également aux artistes qui les ont créés. Michael Lucken prend 
notamment l'exemple du sculpteur Kitamura Seibō, à la fois l'auteur de la «Statue pour la Paix » de Nagasaki, et de celle d'un aviateur militaire, dont une réplique plus grande se trouve à l'entrée du musée (révisionniste) du Yûshûkan, dans l'enceinte du sanctuaire Yasukuni. Ce paradoxe de deux œuvres d'un même artiste illustrant un Japon à la fois victime et héroïque est un bon indicateur de la diversité des mémoires de guerre dans l'archipel.

De la conclusion de l'ouvrage, il faut retenir les trois points suivants. Premièrement, les Japonais n'ont depuis 1945 jamais manifesté d'unité dans leur perception du passé. Il est plus juste à ce propos de parler de discours singuliers, eux-mêmes influencés par des facteurs géographiques. Ce qui peut sembler une évidence doit donc être rappelé : on ne se souvient pas de la guerre dans le Hokkaidō comme à Nagasaki. Deuxièmement, il est impossible, au risque de généraliser, d'opposer comme deux blocs le peuple et les élites. L'État japonais n'a jamais, depuis la fin de la guerre, développé de grand récit national. Troisièmement, il est essentiel d'intégrer l'étude de l'histoire japonaise à celle d'autres aires géographiques. Le comparatisme est un outil des plus intéressants et dont Michael Lucken use à très bon escient, pour montrer les cadres communs comme ses limites. L'insistance de l'auteur et sa reprise des propos d'Arnaud Nanta sur ce point est salutaire, afin d'éviter les jugements européano-centrés et moralisateurs. « Le Japon est infiniment moins étrange quand on l'observe dans sa langue que quand on l'aborde via l'anglais ou le français » (p. 335).

$\mathrm{Au}$ final, Michael Lucken nous offre ici un très bon ouvrage généraliste, dont pourront certainement aussi bénéficier les spécialistes. Le texte est agrémenté de citations variées, rendant la lecture agréable et vivante. Des écrits d'artistes et d'écrivains sont présentés dans chaque chapitre, offrant une perspective nouvelle sur un sujet fréquemment discuté. Original dans son approche, l'auteur insiste à de nombreuses reprises sur la nécessité de dépasser les lieux communs. Les sujets abordés sont vastes et variés, contribuant ainsi à dresser un panorama général de ce que représente la guerre pour les Japonais, d'autant qu'aucun ouvrage complet dédié à cette période n'existait jusqu'alors en langue française. De ce point de vue, le titre Les Japonais et la guerre est fidèle à son contenu et ce livre vient à point nommé combler un vide dans le milieu de la japonologie française.

Bien entendu, aucun ouvrage n'est parfait. Il convient donc de présenter également les limites de celui-ci, à commencer par une vision relativement restreinte du concept d'occupation. Aussi hégémonique qu'ait pu être la présence américaine dans l'archipel, les années 1945-1952 ne peuvent pas être résumées aux seules décisions de Washington. La présence de troupes du Commonwealth britannique dans le sud de Honshū et Shikoku de 1946 à 1951 (avec plus de 40000 hommes et plus de 20000 Japonais travaillant sous leurs ordres, les Australiens ayant notamment pour responsabilité l'assainissement de l'usine de gaz chimique de l'île d'ōkunoshima, dans le département de Hiroshima) représentera au total près d'un quart de toutes les forces d'occupation alliées. Il convient également de rappeler que si l'occupation effective est massivement gérée par les Américains, de larges structures administratives internationales (Far Eastern Commission, Allied Council for Japan) sont également mises en place dans l'archipel, afin de la présenter comme une occupation «alliée » et plurielle. Ces organismes seront amenés à jouer un rôle de soupape important, notamment entre Moscou et Washington alors que les tensions montent entre les deux pays. Ces aspects 
sont autant d'expériences uniques vécues par la population japonaise, qu'il faut également intégrer au tableau général afin de comprendre la période dans sa totalité.

Notons également que si les expériences atomiques de Nagasaki et Hiroshima sont discutées finement par l'auteur, on peut regretter qu'il ne s'attache qu'au discours "standard»sur celles-ci. Seuls les monuments et expériences les plus connus sont présentés dans l'ouvrage. Il aurait été intéressant de ne pas limiter la discussion à ces symboles facilement identifiables, mais également de présenter les rivalités mémorielles en jeu. Pour ne prendre que deux exemples: les anciens travailleurs de l'île d'ōkunoshima, attelés à la fabrication de gaz de guerre qui sera utilisé par l'armée impériale sur le champ de bataille. Atteints de cancers généralisés et de troubles respiratoires après la guerre, leur statut d'agresseur et de victime fut l'objet d'un long combat à l'échelle du département pour obtenir des soins, alors que le nom de Hiroshima sonnait à lui seul comme la quintessence de la souffrance et de l'expérience des armes de destruction massive.

On mentionnera également que la ville haute de Nagasaki, où sont concentrés les monuments dédiés à la bombe, présente une diversité qu'il aurait été judicieux de développer plus avant, à commencer par le « Musée de la Paix » fondé par l'avocat Oka Masaharu. Cette institution (située non loin d'un monument aux travailleurs coréens de Nagasaki) est entièrement dédiée aux victimes coréennes de la bombe et à leur mise en esclavage, aux «femmes de réconfort » ainsi qu'à l'agression japonaise en Asie, et présente un autre aspect de ces mémoires de guerre plurielles au Japon.

20 Toujours à propos des monuments, les discours singuliers sur le passé au Japon dont parle avec raison Michael Lucken sont présentés dans son ouvrage à travers de multiples exemples, mais il aurait également été judicieux de sortir du seul cadre américano-japonais. L'invasion soviétique et la relation entre Moscou et Tōkyō après la guerre ne sont qu'effleurées dans l'ouvrage, au même titre que la monumentalité dédiée à cette question. Certes, il s'agit là d'un sujet mineur en comparaison de l'héritage américain au Japon, mais un regard sur les stèles et les statues dans la ville de Wakkanai (dans le grand nord du Hokkaidō), quasi exclusivement dédiées à la perte de Karafuto (Sakhaline) et à un Japon victime, aurait encore davantage permis de souligner l'importance des mémoires locales, et l'impossibilité d'un discours national commun.

21 Ce choix de ne pas intégrer plus avant la perspective soviétique amène également l'auteur à affirmer que «[...] les Américains ont passé des accords pendant l'occupation avec plusieurs médecins japonais [...] afin qu'ils leur transmettent les données obtenues à partir d'expériences cliniques effectuées sur des prisonniers. En échange, les médecins ne furent pas poursuivis, et il faut attendre le procès de Khabarovsk conduit par les Russes pour que la lumière soit faite sur une partie de ces crimes » (p.216). Il s'agit là d'un fait établi, mais il convient d'ajouter que les Soviétiques firent de même afin de récupérer des données identiques, et c'est pour cette raison que les peines de prison infligées aux coupables furent légères en comparaison des crimes commis.

Le sujet de l'ouvrage étant si vaste, il est toutefois normal que l'auteur n'ait pas pu traiter de tous ces détails en quatre cents pages. Tout ceci rappelle qu'il s'agit d'un excellent ouvrage généraliste. Soutenu par un riche appareil bibliographique, il est parfaitement adapté aux besoins du grand public et intéressera également les spécialistes. Le but est donc atteint pour Michael Lucken qui, on peut en être certain, a 
livré un ouvrage qui constituera une référence dans la japonologie francophone d'aujourd'hui.

\section{NOTES}

1. Igarashi Yoshikuni, Bodies of Memory, Narratives of War in Postwar Japanese Culture, 1945-1970, Princeton, Princeton University Press, 2000.

\section{AUTEUR}

ARNAUD DOGLIA

Université de Genève 